\title{
Theoretical Modeling of Weakly Lensed Polarized Radio Sources
}

\author{
Christopher R. Burns \\ Swarthmore College, Swarthmore, PA 19086 \\ cburns10swarthmore.edu \\ Charles C. Dyer \\ University of Toronto, Toronto Ontario Canada, M5S $3 H 8$ \\ dyer@astro.utoronto.ca \\ Philipp P. Kronberg \\ Los Alamos National Laboratory, IGPP, MS C305, Los Alamos, NM 87501 \\ kronberg@lanl.gov \\ and \\ Hermann-Josef Röser ${ }^{1}$ \\ Max Planck Institut für Astronomie, Heidelberg, Germany \\ roeser@mpia-hd.mpg.de
}

\begin{abstract}
In this paper we present the theoretical basis for the modeling of weakly gravitationally lensed extended sources that are polarized. This technique has been used in the past to constrain the mass profiles of galaxies projected against the jet of the quasar 3C9. Since then, work has been done to improve both the measurement and theoretical modeling of the lensing signal, which manifests itself as an alignment breaking between the morphology and the polarization, parametrized as $\eta_{G}$. To this end, we present the mathematical derivation of the theoretical value of $\eta_{G}$ as well as numerical simulations of expected signals in polarized radio jets. We use the radio jet sources 3C9 and $1253+104$ as illustrative examples of the measurement and modeling of the $\eta_{G}$ signal. For $3 \mathrm{C} 9$, we present constraints on the parameters of the two intervening lenses and quantify their confidence intervals. One lens has no measured redshift and in this case, we show the dependence of mass and mass-to-light ratio on assumed redshift.
\end{abstract}

\footnotetext{
${ }^{1}$ Visiting Astronomer, German-Spanish Astronomical Centre, Calar Alto, operated by the Max-Planck-Institute for Astronomy, Heidelberg, jointly with the Spanish National Commission for Astronomy.
} 
Subject headings: gravitational lensing — polarization — galaxies: fundamental parameters - galaxies: jets — quasars: individual (3C9, QSO1253+104)

\section{INTRODUCTION}

The action of a gravitational lens is to map the source plane to the image plane. This mapping can either be one-to-many (multiple images) or one-to-one (weak lensing). In the majority of cases, we do not know the structure of the source plane and must make some assumptions about it in order to invert the lens equation and solve for the lens parameters. However, if the source is extended and polarized, then we can have an undistorted view of the structure of the unlensed source plane since the polarization, being a one-point vector, is unchanged by a gravitational lens (Kronberg et al. 1991; Dyer and Shaver 1992). The morphology, however, is distorted due to the differential mapping of different points in the source plane, which represent a family of 2-point vectors. The fact that a source is polarized indicates a preferred direction in the source plane which is often aligned with, or physically coupled to, the morphology. Astrophysical jets are believed to originate from strong magnetic fields which accelerate charged particles and produce the synchrotron radiation that makes the jet's morphology visible (Honda and Honda 2002; Colgate et al. 2001; Benford 1978). It is therefore expected that the polarization should be aligned with the jet direction and this is observed to be the case (Bridle et al. 1986).

A key advantage of this method is that it provides the possibility of uniquely probing the global mass and mass distribution of a single galaxy, rather than by statistical methods that use collections of individual galaxy lenses. The polarimetric properties of radio sources provide unique advantages for this (see below), one being that the linear scales of extragalactic radio source jets are comparable with a single galaxy's mass scale. The method could measure all the mass included within a galactocentric radius, $r \lesssim 100 \mathrm{kpc}$.

A disadvantage is that the "setup" for this type of single-galaxy mass probe requires some serendipity in getting advantageous lens-source alignments, examples of which we discuss in this paper. Up to now these are generally few, being limited by the sensitivity of the immediate past generation of large optical telescopes. For the ideal galaxy mass probe, quantitative light profiles of the lensing galaxy, with colors, on scales comparable with the background radio jet images would give estimates of the mass-to-light ratio $(\mathcal{M} / \mathcal{L})$. This sort of detail will become possible with the future NGST optical, and EVLA radio telescopes, and more powerful ground-based optical telescopes. It is therefore timely at this point to examine the lensing theory aspects for radio sources, which have yet to come to full fruition, but are potentially very powerful single-galaxy mass probes.

A source can be characterized by two vector fields: its morphology defined by Stokes parameter I, and that by the linear polarization (Stokes Q and U). The morphological features will be mapped as two-point vectors and will rotate. Therefore, if the two vector fields were aligned in the source 
plane, any differential gravitational lensing will cause the breaking of this alignment by an angle we call $\eta_{G}$. This has been measured and used to constrain the mass of galaxy-scale lenses in front of the jet of quasar 3C9 (Kronberg et al. 1991, 1996). More generally, it could be used to measure the gravitational lensing of any source where morphology and polarization are intrinsically correlated in the source plane.

In the following sections, we present the theoretical derivation of the alignment breaking parameter $\eta_{G}$ for the case of a linear morphology applicable to astrophysical jets and the peripheral boundaries of radio lobes. We follow with numerical simulations for an ideal jet to illustrate the measurement and modeling of the alignment breaking. We then apply these new techniques to the quasar 3C9 and derive masses and mass-to-light ratios for the two intervening galaxies. Lastly, we present the observations and analysis of the quasar $1253+104$, which shows no alignment breaking and is consistent with the observed redshift of the intervening galaxy.

\section{DERIVATION OF $\eta_{G}$}

In order to measure the $\eta_{G}$ signal, one must obtain representative vectors which define the local morphology of the source. In the case of astrophysical jets, which are typically one-dimensional (i.e., the transverse angular size of the jet is much less than its linear extent or even the resolving power of the telescope), the tangent vector is used. We therefore ascribe a curve $\boldsymbol{\Gamma}(\theta)$, which we call the fiducial curve, which best defines the path of the jet or lobe boundary (see Figure 1). At each point, one can then measure the tangent vector $\boldsymbol{\Psi}(\theta)=d \boldsymbol{\Gamma} / d \theta$ and the intrinsic polarization angle $\chi(\theta)$. For an ideal alignment and no gravitational lensing, one expects the angle of the tangent vector to be aligned with the polarization $(\psi(\theta)=\chi(\theta)$, where $\psi$ is the angle of the tangent vector). In practice, it is more likely there is some intrinsic deviation from the ideal case and we therefore should write $\chi(\theta)=\psi(\theta)+\kappa(\theta)$, where $\kappa$ represents any intrinsic deviation from perfect alignment in the source plane. With these definitions in hand, the alignment breaking parameter is defined (Kronberg et al. 1991):

$$
\eta_{G}=\chi(\theta)-\psi(\theta)+\kappa(\theta)
$$

such that in the ideal case with no gravitational lensing, we expect $\eta_{G}=0$ over the length of the structure.

It is convenient to think of the projected positions of the lenses and sources on the sky as complex numbers. Following the complex notation of Bourassa et al. (1973), we label image-plane positions as $\mathbf{r}$ and the source-plane positions as $\tilde{\mathbf{r}}$. The action of a gravitational lens is therefore to map each source-plane point to one or more points on the image plane. This map can be written as

$$
\tilde{\mathbf{r}}=\mathbf{r}-\sum_{l} D_{l} \mathbf{I}_{l}^{*}(\mathbf{r})
$$

where summation is over each lens along the line of sight, $D_{l}$ is the distance factor, $\mathbf{I}_{l}(\mathbf{r})$ is the complex scattering function and the asterisk denotes complex conjugation (see Bourassa et al. 
(1973)). In the case of spherically symmetric potentials, equation (2) simplifies to:

$$
\tilde{\mathbf{r}}=\mathbf{r}-\sum_{l} D_{l} \frac{\tilde{m}\left(\left|\mathbf{r}-\mathbf{r}_{l}\right|\right)\left(\mathbf{r}-\mathbf{r}_{l}\right)}{\left|\mathbf{r}-\mathbf{r}_{l}\right|^{2}}
$$

where $\tilde{m}(r)$ is the cylindrical mass, defined as the total mass contained in a cylinder centered on the lens and with radius $r$ equal to the impact parameter (Dyer 1977). Depending on the structure of the mass profiles and the magnitude of $D_{l}$, equations (2) and (3) will have either one or an odd number of solutions, corresponding to the strong and weak lensing regimes.

Using equations (1) and (2), one can formalize the value of $\eta_{G}$ for an ideal jet (i.e., $\left.\kappa(\theta)=0\right)$. Let $\boldsymbol{\Psi}(\theta)$ be the tangent vector to the path taken by the jet in the image plane, defined by $\boldsymbol{\Gamma}(\theta)$ $(\boldsymbol{\Psi}(\theta)=d \boldsymbol{\Gamma} / d \theta)$. Likewise, let $\tilde{\boldsymbol{\Psi}}(\theta)$ and $\tilde{\boldsymbol{\Gamma}}(\theta)$ be the tangent vector and the path in the source plane (see Figure 1). Following equation (2), we can write

$$
\tilde{\boldsymbol{\Gamma}}(\theta)=\boldsymbol{\Gamma}(\theta)-\sum_{l} D_{l} \mathbf{I}_{l}^{*}(\boldsymbol{\Gamma}(\theta))
$$

We assume that $\chi(\theta)=\tilde{\psi}(\theta) \equiv \arg (\tilde{\Psi})$ (i.e., perfect alignment between morphology and intrinsic polarization) and by differentiating equation (4), we obtain

$$
\tilde{\mathbf{\Psi}}(\theta)=\mathbf{\Psi}(\theta)-\sum_{l} D_{l}\left[\frac{\partial \mathbf{I}_{l}^{*}}{\partial x} \frac{d x}{d \theta}+\frac{\partial \mathbf{I}_{l}^{*}}{\partial y} \frac{d y}{d \theta}\right]
$$

where we have taken $\boldsymbol{\Gamma}(\theta)=x(\theta)+i y(\theta)$. With this notation, the tangent vector can be written as $\boldsymbol{\Psi}(\theta)=d x / d \theta+i d y / d \theta$ and the tangent angle is $\psi=\arg (\boldsymbol{\Psi})$. Substituting this into equation (1), we get the result:

$$
\begin{aligned}
\eta_{G}(\theta)= & \arg \left\{\frac{d x}{d \theta}-\sum_{l} D_{l}\left(\frac{\partial R e(\mathbf{I})}{\partial x} \frac{d x}{d \theta}+\frac{\partial R e(\mathbf{I})}{\partial y} \frac{d y}{d \theta}\right)\right. \\
& \left.+i \frac{d y}{d \theta}+i \sum_{l} D_{l}\left(\frac{\partial \operatorname{Im}(\mathbf{I})}{\partial x} \frac{d x}{d \theta}+\frac{\partial \operatorname{Im}(\mathbf{I})}{\partial y} \frac{d y}{d \theta}\right)\right\}-\psi
\end{aligned}
$$

Switching to component notation, equation (6) can be cast in a more compact form:

$$
\eta_{G}(\theta)=\arg \left\{\sigma^{i j}(\theta) \psi_{j}(\theta)\right\}-\psi
$$

were $\psi_{j}(\theta)=(d x / d \theta, d y / d \theta)$ and $\sigma^{i j}$ is the shear matrix:

$$
\sigma^{i j}=\left[\begin{array}{cc}
1-\sum_{l} D_{l} \frac{\partial R e(\mathbf{I})}{\partial x} & -\sum_{l} D_{l} \frac{\partial R e(\mathbf{I})}{\partial y} \\
\sum_{l} D_{l} \frac{\partial \operatorname{Im}(\mathbf{I})}{\partial x} & 1+\sum_{l} D_{l} \frac{\partial \operatorname{Im}(\mathbf{I})}{\partial y}
\end{array}\right]
$$

From this, we see that $\eta_{G}$ is a measure of the differential bending projected along the tangent vector of the jet. From equation (7), we see that $\eta_{G}=0$ if $\psi_{j}$ is an eigenvector of $\sigma^{i j}$. In the case of a single, spherically symmetric lens, this corresponds to radial and tangential vectors. We 
can therefore imagine pathological cases where a gravitational lens may be present, yet one does not detect any signal. For example, in the case where the jet follows a straight, radial path away from the lens, the differential bending is parallel to the tangent vector everywhere on the jet and $\eta_{G}$ is therefore zero. Another, more unlikely case is where the jet "circles" a spherically symmetric lens at a constant impact parameter. In this case, the differential bending is zero along the jet and $\eta_{G}$ is again zero.

\section{SIMULATIONS}

The purpose of simulating the weak lensing of an ideal jet is twofold: (1) to determine the best way to objectively choose the fiducial line and (2) to test the sensitivity of $\eta_{G}$ to the various lens parameters. For our testbed, we construct a fake jet $15^{\prime \prime}$ long by convolving a straight line with a Gaussian beam having FWHM of $3^{\prime \prime}$, which is typical of our observations. This fake jet can then be lensed with any mass distribution desired. We choose a spherically symmetric King profile, whose density is given by

$$
\begin{aligned}
& \rho(r)=\rho_{o}\left(1+(r / a)^{2}\right)^{-3 / 2}, r \leq r_{c} \\
& \rho(r)=0, r \gtrsim r_{c}
\end{aligned}
$$

where $a$ is the core-radius, the radius at which the density falls to about $35 \%$ of its central value $\left(\rho_{o}\right)$ and $r_{c}$ is the cut radius. Unless otherwise stated, all the modeling in this paper will be done with this potential. We use this lensed jet to first test our method for determining the fiducial line, $\boldsymbol{\Gamma}(\theta)$.

Initial values of $\boldsymbol{\Gamma}(\theta)$ are computed by taking a weighted average of the jet's isophotal contours. This typically results in an $\eta_{G}$ signal which shows the correct sinusoidal shape as well as the expected small scale random variations due to badly chosen jet points (Kronberg et al. 1996). The $\eta_{G}$ signal has a scale comparable to the angular scale of the lens itself and therefore has a large radius of curvature. Small-scale variations represent effects that are not gravitationally induced and are recognized by their small radii of curvature. To correct these errors, the jet points responsible for the variations are located and varied by a small amount perpendicular to the jet axis in an attempt to reduce the small-scale curvature of the $\eta_{G}$ signal. The gravitationally induced $\eta_{G}$ signal will not be eliminated by varying the position of just one jet point. Figure 2 shows the measured value of $\eta_{G}$ for a lensed jet before and after this correction is performed.

We now proceed to model the weak lensing and extract the parameters. The downhill simplex method (Press et al. 1992) is used to find the best fit by minimizing the $\chi^{2}$ statistic. Assuming that the angular position and redshift of each lens has been measured, there are only 4 remaining parameters to be fitted: the total mass $M_{G}$ probed by the lens, the mass profile function $\rho(r)$, the core radius $a$ and the cut radius $r_{c}$. One should be mindful of the shape of the parameter space and we now turn to simulations of $\chi^{2}$ to attempt to ascertain the degeneracies in the parameters. 
We begin with the cutoff radius, $r_{c}$. This is only relevant to the mass models whose profiles do not converge to a finite mass as $r \rightarrow \infty$. One might expect that as the cutoff radius is increased, the best fit value for the total mass will increase so as to maintain enough mass at smaller radii to reproduce the observed lensing. Figure 3 shows the value of $\chi^{2}$ as a function of the model mass and cut-off radius. Indeed, in the middle impact parameter zone $\left(20 \lesssim r_{c} \lesssim 60 \mathrm{kpc}\right)$, larger cut-off radii require larger masses. We note however that $\chi^{2}$ becomes independent of $r_{c}$ for $r_{c} \lesssim 20 \mathrm{kpc}$ because the cut-off radius is now less than the smallest impact parameter, which we shall denote as $R_{i}$ of the lens-jet system (see Figure 1). It also becomes less sensitive to $r_{c}$ for $r_{c} \gtrsim 60 \mathrm{kpc}$, which is comparable to the furthest impact parameter $\left(R_{o}\right)$ for the jet-lens system. This is due to the fact that as the cut-off radius is increased, the additional mass outside $R_{o}$ acts more like a mass-sheet, to which $\eta_{G}$ (and any other weak-lensing analysis) is increasingly insensitive. It is therefore not worth investigating values of $r_{c} \gg R_{o}$.

Figure 4 shows the value of $\chi^{2}$ as a function of the mass and core radius for a King profile lens at two different distances from the jet. Clearly, in the first case, the galaxy's mass is well constrained by the modeling; however, the core radius is not so well constrained. This is because the jet probes radii ranging from $R_{i}=20 \mathrm{kpc}$ to $R_{o}=60 \mathrm{kpc}$ and is therefore relatively insensitive to changes in the internal structure (i.e., $R \lesssim 20 \mathrm{kpc}$ ) of the lens. If one also imposes the constraint that the lens be incapable of producing multiple images, then a lower limit can be placed on the core radius, as shown by the dashed line in the first panel of Figure 4. If the jet probes smaller radii of the lens potential, the modeling will be able to better constrain the core radius, as shown the second panel of Figure 4, where the lens has been moved closer $\left(1^{\prime \prime}\right)$ to the jet such that $R_{i}=10 \mathrm{kpc}$.

The mass profile function is usually not well constrained by the modeling, except in extreme cases such as the de Vaucouleurs exponential profile where the core is sharp enough that one cannot get enough mass interior to $R_{i}$ and $R_{o}$ without causing the jet to be multiply imaged. This case is

shown in Figure 5. Note that all the $\chi^{2}$ contours lie under the minimum core radius curve (dashed line).

\section{MODELING OF 3C9}

The quasar 3C9 $(z=2.012)$ was the first object analyzed with the alignment breaking technique. The first panel of Figure 6 shows the radio emission and polarization vectors for 3C9 as well as the the fiducial line defining the jet's morphology. For clarity, we plot the magnetic field component of the polarization, which is simply rotated $90^{\circ}$ with respect to the electric field component ${ }^{1}$. It is an interesting case in that initially, only one lens (a galaxy at $z=0.2538$ ) was in evidence (see

\footnotetext{
${ }^{1}$ In their original definition of $\eta_{G}$, Kronberg et al. (1991) explicitly subtract $90^{\circ}$ from the observed polarization for this reason.
} 
Kronberg et al. 1991). However, the impact parameter and tangential position made it difficult to model the observed $\eta_{G}$ with only one galaxy and this led the authors to obtain deeper images of the field. The result was the detection of a fainter galaxy with a smaller impact parameter (see Kronberg et al. 1996). While most likely less massive, the latter galaxy provides a better fit to the observed values of $\eta_{G}$ due to its small impact parameter. The fact that the fainter galaxy was first detected gravitationally demonstrates the power of our technique. Unfortunately, it was not possible to directly obtain a spectroscopic redshift for the fainter galaxy.

The plot for the observed values of $\eta_{G}$ along the jet is shown in Figure 7 . The error bars are computed by adding the intrinsic scatter of the polarization and the uncertainty in choosing the jet points, which are determined by varying their positions by $0.1^{\prime \prime}$ perpendicular to the axis of the jet. There is a clear variation of $\eta_{G}$, as is expected from the location of the lenses.

Even though the lens positions have been measured, it is instructive to measure $\chi^{2}$ as a function of lens position to see whether or not the $\eta_{G}$ signal itself points to the likely location of the lens in the absence of a detection of the galaxies. We choose a King profile with mass $10 \times 10^{11} M_{\odot}$ and ensure that there are no multiple images produced, consistent with observations. We then compute $\chi^{2}$ for putative positions of a single lens over a search area on the sky. Figure 8a shows the results. Note that there are two preferred locations for minimizing $\chi^{2}$ : to the east and to the west. In this case, the best fit actually occurs to the east of the quasar jet, whereas the observed lens is to the west, close to the second minimum. Both these positions are favored because they tend to straighten the jet in the source plane. However, if we increase the mass, the minimum $\chi^{2}$ shifts to the west and the eastern minimum all but disappears. This is shown in Figure 8b. The reason the minimum $\chi^{2}$ does not correspond exactly to the location of G2 is because it is sensitive to sum of the masses of both lenses and G1 in effect "drags" the minimum a little to the east.

Given that the positions of the two lenses in the field of 3C9 are observationally determined, we can allow the other parameters to vary in this "galaxy mass laboratory". An obvious combination to explore is the masses of both lenses. We therefore compute $\chi^{2}$ for different combinations of masses for galaxy 1 and 2 (G1 and G2). Because G2 has no measured redshift, we cannot provide an upper bound to its mass. However, a lower limit to its mass is determined by fixing its redshift at $z_{2} \simeq 0.25$. This corresponds to the maximum of the distance factor. However, since the computed absolute luminosity of the galaxy is also a function of redshift, $z_{2}=0.25$ does not yield the minimum $\mathcal{M} / \mathcal{L}$ (it would have to be in excess of several thousand $M_{\odot} / L_{\odot}$ ). Given this uncertainty, we compute $\chi^{2}$ letting the redshift and mass vary to find the minimum mass of G2 consistent with the data. The results are shown in Figure 9a. We then do the same exercise, but now letting the redshift and $\mathcal{M} / \mathcal{L}$ for galaxy 2 vary, the results of which are shown in Figure $9 \mathrm{~b}$. Here, we have used an apparent magnitude of $m_{R}=23$ (Kronberg et al. 1996). K-corrections for an Sbc galaxy are given in Coleman et al. (1980), and we assume an Einstein-deSitter cosmology ${ }^{2}$. As predicted,

\footnotetext{
${ }^{2}$ We choose this cosmological model in order to be consistent with Kronberg et al. (1996) with $H_{o}=75$. For the redshifts of interest in this paper, the results are quite insensitive to $\Omega_{m}$ and $\Omega_{\Lambda}$.
} 
the minimum mass occurs at $z \simeq 0.25$ and is approximately $10 \times 10^{11} M_{\odot}$. However, examining the behavior of $\mathcal{M} / \mathcal{L}$ with redshift, a low $\mathcal{M} / \mathcal{L}$ is favored by a lens with a redshift in the range $\left(1 \lesssim z_{2} \lesssim 1.8\right)$, where its value $\mathcal{M} / \mathcal{L} \simeq 20$ is largely insensitive to changes in redshift. Interestingly, there is an absorption feature in 3C9's spectrum at a redshift of $z_{a b s}=1.6$ (Kronberg et al. 1996). This would, however, require a mass for G2 on the order of $6 \times 10^{12} M_{\odot}$ and R-band luminosity of $2 \times 10^{11} L_{\odot, R}$.

The smallest impact parameter between the jet and G2 is approximately 2 arc-seconds which at a redshift $z \simeq 1$ corresponds to a physical distance of $12 \mathrm{kpc}$. We therefore find that the best fit $\chi^{2}$ is insensitive to the core radius, as discussed in $\S 3$. We can, however, use the fact that there are no observed secondary images of the quasar or jet to place lower limits on the core radius of G2, provided its redshift is assumed. For both $z_{2}=1.0$ and $z_{2}=1.6$, we establish a minimum core radius of $5 \mathrm{kpc}$.

Unlike G2, the redshift of G1 has been measured and one can place confidence limits on its mass. By varying the mass of G1 and G2 around the best fit parameters, we get the $\chi^{2}$ contours shown in Figure 10, where we have set $z_{2}$ to be the two most likely redshifts for G2 $\left(z_{2}=1.0\right.$ and $z_{2}=1.6$ ). Clearly, the mass of G2 is more tightly constrained than G1 (when the redshift is fixed), since G2 is closer to the jet. Note that the redshift and mass of G2 do not affect the best estimate of G1 and so we find that G1 is "decoupled" from the parameters of G2, as noted in Kronberg et al. (1996).

Figure 11 shows the best fit as well as two typical fits corresponding to the $1 \sigma$ confidence interval. None of our models can reproduce the very sharp decrease of $\eta_{G}$ at $\theta>6^{\prime \prime}$. In this particular system, the "sharpness" of the model $\eta_{G}$ curve is largely a function of the projected distance of the galaxy G2 from the jet and is fixed by observation. However, the region $\theta>6^{\prime \prime}$ is where the jet makes a sharp turn and rapidly decreases in brightness (see Figure 6). It may be that this is the transition region between the jet and lobe, where the assumption of alignment between jet morphology and polarization breaks down. Nevertheless, our models do reproduce the $\eta_{G}$ behavior in the region $3<\theta<6^{\prime \prime}$, where the signal is highest and the jet morphology is unambiguous.

Table 1 summarizes the parameters derived from the preceding analysis. For the purposes of comparison, we list the best fits for a variety of redshifts for G2. The last row gives results when no constraints are placed on $z_{2}$ and so should be taken as the firm limits. In this case, the only parameter which can be fully constrained is the mass of G1 out to approximately 10 arc-seconds $(r \simeq 30 \mathrm{kpc}$ at $z=0.25)$. The confidence intervals were determined by projecting the $1 \sigma \chi^{2}$ surface onto the axis of the parameter in question. For the joint probability distribution of $M_{G 1}$ and $M_{G 2}$, this corresponds to $\Delta \chi^{2}=2.3$, whereas the confidence interval for $M_{G 1}$ only (last row of Table 1) corresponds to $\Delta \chi^{2}=1$. The masses quoted for G2 are out to approximately 6 arc-seconds, which corresponds to $r \simeq 35 \mathrm{kpc}$ at redshifts $z \simeq 1$. Given that our estimates for the mass of G2 at each redshift are for $r \lesssim 6$ arc-seconds, well beyond the observed light of G2, we give $(\mathcal{M} / \mathcal{L})_{G 2}$ as upper 
limits.

\section{QSO1253+104: OBSERVATIONS}

QSO1253+104 is a classic FRII radio source at a redshift of $z=0.83$. The jets extend to the North-West and to the South-East approximately $10^{\prime \prime}$ in each direction. Both jets show extended structure, especially the North-West jet/lobe. Figure 6 shows the radio contour map at $5 \mathrm{GHz}$. The data was taken at the VLA as part of the $\eta_{G}$ survey conducted by Kronberg et al. The first deep optical image of the field in the literature is due to Hutchings (1992), in which 3 objects are visible within $30^{\prime \prime}$ of the QSO.

A sample of distant quasars with large scale jets was observed since June 1993 during several runs on the 3.5m-telescope on Calar Alto using FREDUK and MOSCA. The vicinity of the jets was searched for intervening galaxies which would cause "alignment breaking". QSO1253+104 was imaged in B, R and I on 1997 February 10 with MOSCA using a $2 \mathrm{k} \times 2 \mathrm{k}$ Loral CCD with $15 \mu$ pixels corresponding to 0.32 arcsec on the sky, following the earlier detection of a candidate galaxy with FREDUK in June 1994. Figure 12 shows the sum of three $600 \mathrm{sec}$ images in the R-band.

Spectra of the galaxy labled G in Figure 12 were taken with MOSCA on 2000 February 9 and 10. In that run MOSCA was equipped with a SITe CCD of $2 \mathrm{k} \times 4 \mathrm{k} 15 \mu$ pixels. Grism green-500 was used together with an order separation GG495 filter. The dispersion was $2.75 \AA$ per pixel covering the wavelength range 5500 to $9200 \AA$. For a rough flux calibration the standard star HZ 2 was observed. Three separate spectra with $3000 \mathrm{sec}$ integration time were taken. The analysis of the spectra was carried out with context long in MIDAS. Figure 13 shows the reduced and summed spectrum. A prominent emission line is seen at a wavelength of $6527.0 \AA$. This line was already detected with spectra taken with the same instrument in February 1997 and June 1998, although the quality of those spectra was inferior to the ones presented here.

The line cannot be due to [OIII]4959,5007 as we do not see the other line of this duplet. If it is due to [OII] 3727 we should see the [OIII] lines in the red wavelength range around $8700 \AA$. We have taken two $3000 \mathrm{sec}$ exposures with the red-1000 grating covering the range from 5000 to above $9000 \AA$. Unfortunately this region is heavily contaminated with night sky lines, so we cannot exclude that the line seen in the green-500 spectrum is indeed [OII] 3727. This would then argue

for a redshift of 0.75 for the galaxy. $\mathrm{H} \beta$ at $8513 \AA$ is, however, also not seen. No significant lines were seen in the blue spectral range observed with the blue-500 grating in two 3000 sec exposures.

\section{MODELING OF 1253+104: THE NULL RESULT}

Figure 14 shows the optical field of $1253+104$ with the radio emission drawn as contours. The sources to the South-West are closely aligned with the jet axis and would produce very little $\eta_{G}$ 
signal. The sources to the North-East and North-West, however, are off-axis and close enough to be able to produce an $\eta_{G}$ signal. However, the sources to the east are most likely stellar and the extended source to the North-West is likely at a redshift of 0.75 (see $\S 5$ ), which is very close to the quasar's redshift.

Figure 6 shows the North-East jet, its polarization structure and the choice of the fiducial line which was computed by averaging the intensity contours. One can clearly see that the polarization follows the morphology quite closely. Using the same methods described above, we compute $\eta_{G}$, which is plotted in Figure 7. As one can see, the $\eta_{G}$ signal is very weak and, in fact, consistent with zero to within the error bars.

We conclude that there is no detectable gravitational lensing in the jet of QSO1253+104. This is entirely consistent with the fact that the only viable lens was found to be at a comparable redshift to that of the radio jet, so that $D_{l} \simeq 0$ (see equation (7)). While this constitutes a null result, it is still an important null result, in that it establishes that the morphology and polarization do indeed follow one another. We can also be more confident that our method of determining the uncertainty in $\eta_{G}$ is appropriate, since our initial measurement produced a signal that was consistent with zero to within the uncertainties.

\section{COMMENTS}

The observed $\eta_{G}$ is very sensitive to the choice of representative points along the jet. Furthermore, once the jet points are determined, they remain fixed for all subsequent modeling, so it is worth investing some time in determining the "best" representative points. Through simulations, we find that using the average behavior of the isophotal contours followed by a curvature minimization algorithm consistently reproduces reasonable values of $\eta_{G}$. This methodology requires very little human intervention and is therefore more objective than choosing jet points by eye.

In the most favorable situation where all lenses are identified and their positions in angular and redshift space are measured, $\eta_{G}$ can yield good estimates of the mass of a galaxy that is interior to the largest impact parameter $R_{o}$. One important advantage of the $\eta_{G}$ technique is that it measures a continuous range of impact parameters for the lens, ie., a jet can probe both large and small impact parameters. Therefore, in principle one can also directly measure the mass profile of individual galaxies. We also find that the ellipticity of the lens's mass distribution usually has very little effect on $\eta_{G}$ and can therefore be neglected in the determination of the lens parameters.

\section{CONCLUSIONS}

The $\eta_{G}$ technique is a useful astrophysical tool for measuring the mass of galaxy-sized gravitational lenses. With the more than 600 quasars and AGN known to have radio jets (Liu and Zhang 
2002), we have an excellent laboratory in which to measure the physical parameters of advantageously placed intervening galaxies. The numbers of measurable, individual mass intervenors will improve with the next generation of radio and optical telescopes. With a large enough sample, one could in principle begin to constrain the relevant cosmological parameters using statistical methods (see Falco et al. (1998); Keeton (2002)).

In the case of $3 \mathrm{C} 9$, we have used a more statistically robust method to find the best fit lens parameters of two intervening galaxies and place confidence intervals on their values. For galaxy G1, we recover the results of Kronberg et al. (1996): total mass of $17 \pm 10 \times 10^{11} M_{\odot}$ and an upper limit of 75 for the mass-to-light ratio. For galaxy G2, which has no measured redshift, we can place an overall lower limit on the mass: $10 \times 10^{11} M_{\odot}$, corresponding to a redshift of $z_{2}=0.25$. However, this would lead to an unrealistic mass-to-light ratio and we conclude that it is more likely the galaxy has a redshift $z_{2} \gtrsim 1$. If one identifies this galaxy with the absorption feature at $z_{a b s}=1.6$, this leads to $M_{G 2}=65 \pm 15 \times 10^{11} M_{\odot}$ and $\mathcal{M} / \mathcal{L} \lesssim 30$. Also, due to the observed lack of multiple images, we can place a lower limit on the core mass of G2: $a_{G 2}>5 \mathrm{kpc}$.

While it is important to find as many gravitational lenses as possible, establishing null results like QSO $1253+104$ is also important. In this way, we can determine a statistical "baseline" of the intrinsic, non-gravitational deviations between the morphology and polarization $(\kappa)$ and hence better determine the level of significance of any detections of gravitational lensing.

The quasar 3C9 provides a good example of the methodology we have developed and illustrates the strengths and limitations of the technique. QSO 1253+104 provides a useful null result in that our methodology correctly measures an $\eta_{G}$ consistent with no gravitational lensing.

We especially thank the director and staff of the Calar Alto Observatory and the Max-PlanckInstitut für Astronomie, Heidelberg for their hospitality, and for the generous allotment of time on the Calar Alto 3.5m. telescope. CRB, CCD and PPK wish to acknowledge the Natural Sciences and Engineering Research Council of Canada for support through their post-graduate scholarship (CRB) and research grant programs (CCD and PPK). Finally, we wish to thank the anonymous referee for constructive comments and Margaret Burbidge for assistance at early stages of this project. 


\section{REFERENCES}

Benford, G. 1978, MNRAS, 183, 29

Bourassa, R. R., Kantowski, R., \& Norton, T. D. 1973, ApJ, 185, 747

Bridle, A. H., Perley, R. A., \& Henriksen, R. N. 1986, AJ, 92, 534

Coleman, G. D., Wu, C. C., \& Weedman, D. W. 1980, ApJS, 43,393

Colgate, S. A., Li, H., \& Pariev, V. 2001, Physics of Plasmas, 8, 2425

Dyer, C. C. 1977, MNRAS, 180, 231

Dyer, C. C. \& Shaver, E. G. 1992, ApJ, 390, L5

Falco, E. E., Kochanek, C. S., \& Munoz, J. A. 1998, ApJ, 494, 47

Honda ,M., \& Honda ,Y. S. 2002, ApJ, 569, L39

Hutchings, J. B. 1992, AJ, 104, 1311

Keeton, C. R. 2002, ApJ, 575, L1

Kronberg, P. P., Dyer, C. C., Burbidge, E. M., \& Junkkarinen, V. T. 1991, ApJ, 367, L1

Kronberg, P. P., Dyer, C. C., \& Roeser, H. J. 1996, ApJ, 472, 115

Liu, F. K. \& Zhang, Y. H. 2002, A\&A, 381, 757

Press, W. H., Teukolsky, S. A., Vetterling, W. T., \& Flannery, B. P. 1992, Numerical Recipes in C (2d ed.; Cambridge: Cambridge Univ. Press) 


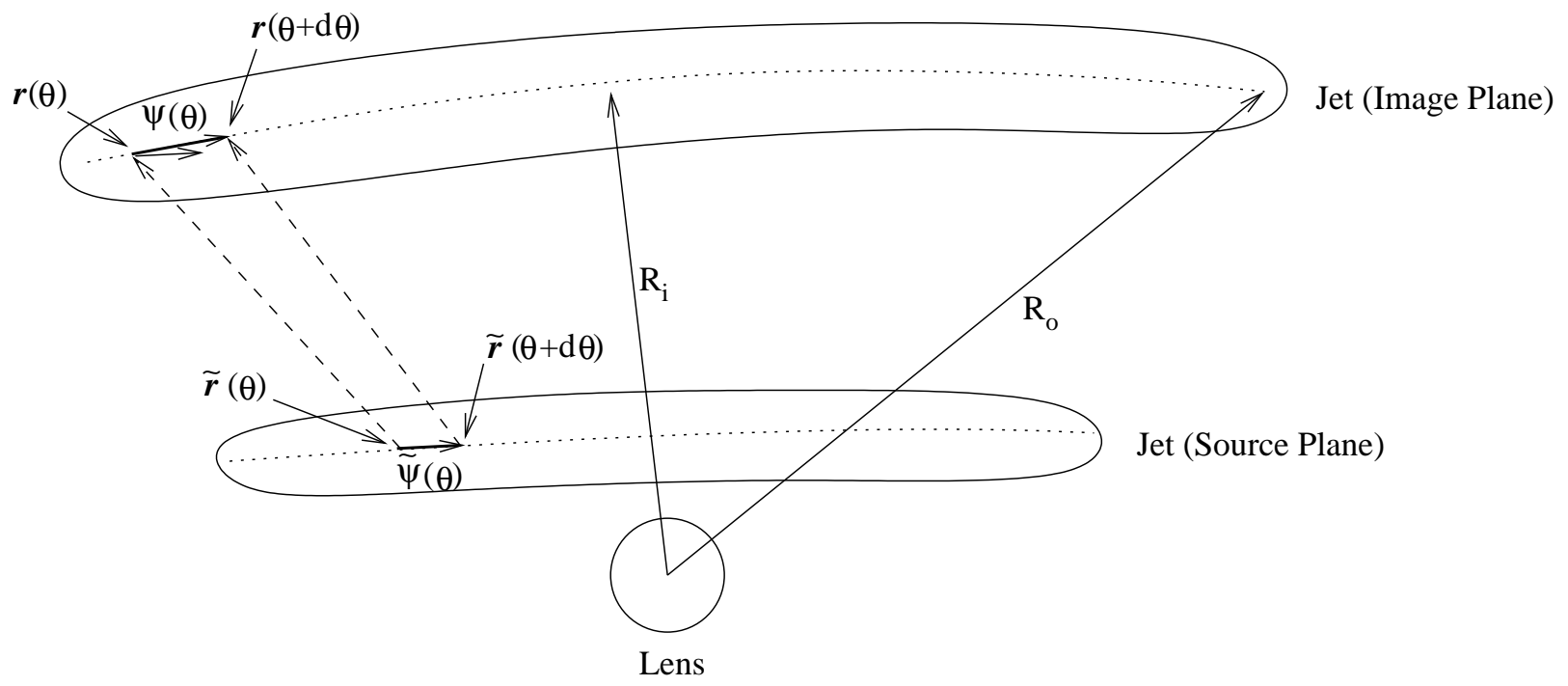

Fig. 1. - The lensed (image plane) and unlensed (source plane) images of a source with one dimensional morphology. The dotted lines represent the fiducial lines. The dashed lines show the mapping between two points in the source plane and their corresponding points in the image plane. Note that the tangent vector in the source plane $(\tilde{\boldsymbol{\Psi}})$ has been reproduced below the tangent vector in the image plane $(\boldsymbol{\Psi})$ to illustrate the alignment breaking. The minimum and maximum impact parameters probed by the jet $\left(R_{i}\right.$ and $R_{o}$, respectively) are drawn with arrows. 


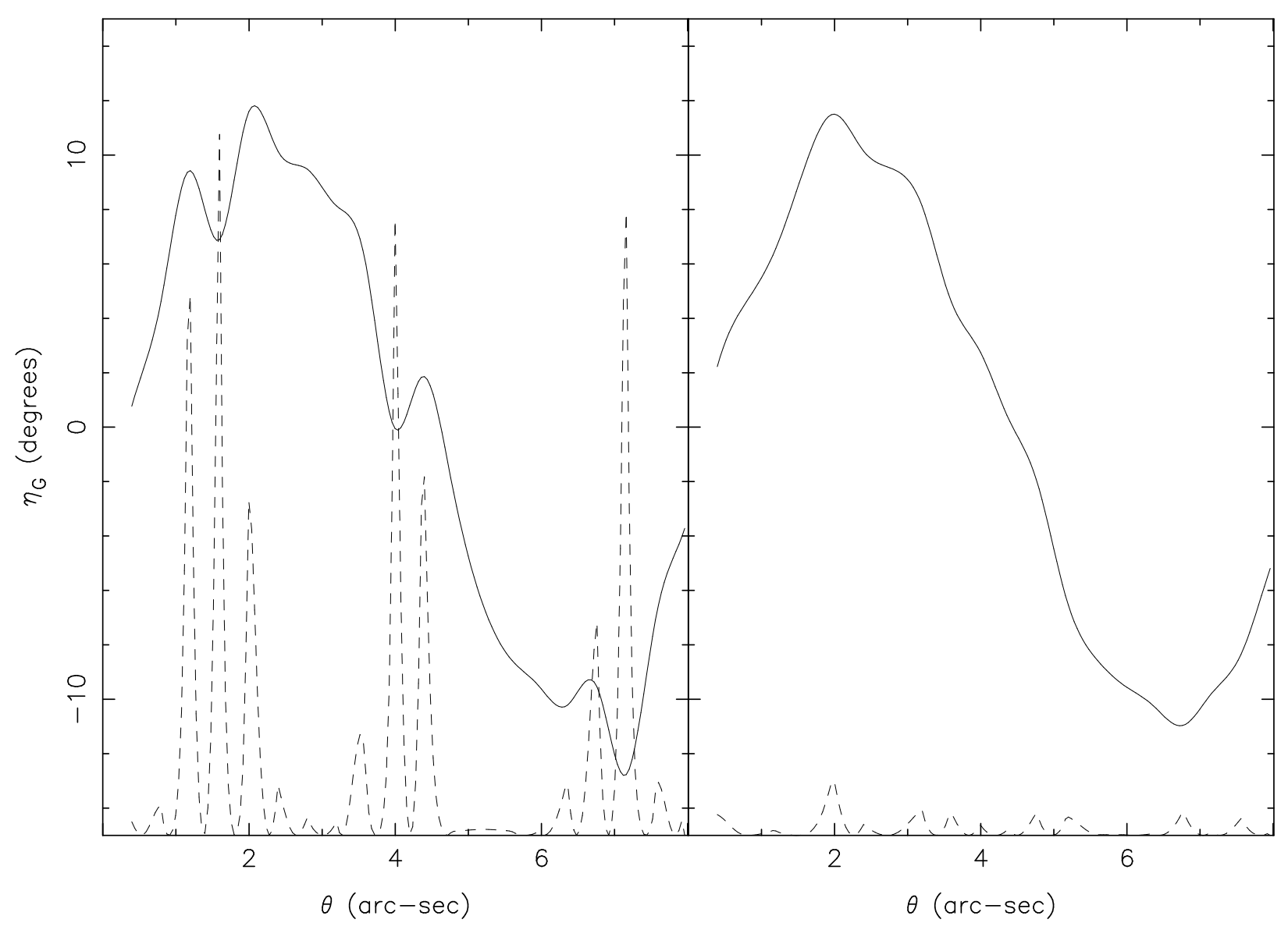

Fig. 2.- Plot of $\eta_{G}$ using only the contour averaging algorithm (first panel) and the contour averaging followed by the curvature minimization algorithm after 7 iterations (second panel). In both cases, the dashed line is proportional to the curvature (or inversely proportional to the radius of curvature) of $\eta_{G}$ along the jet. The horizontal axis represents the distance along the jet in arc-seconds. 


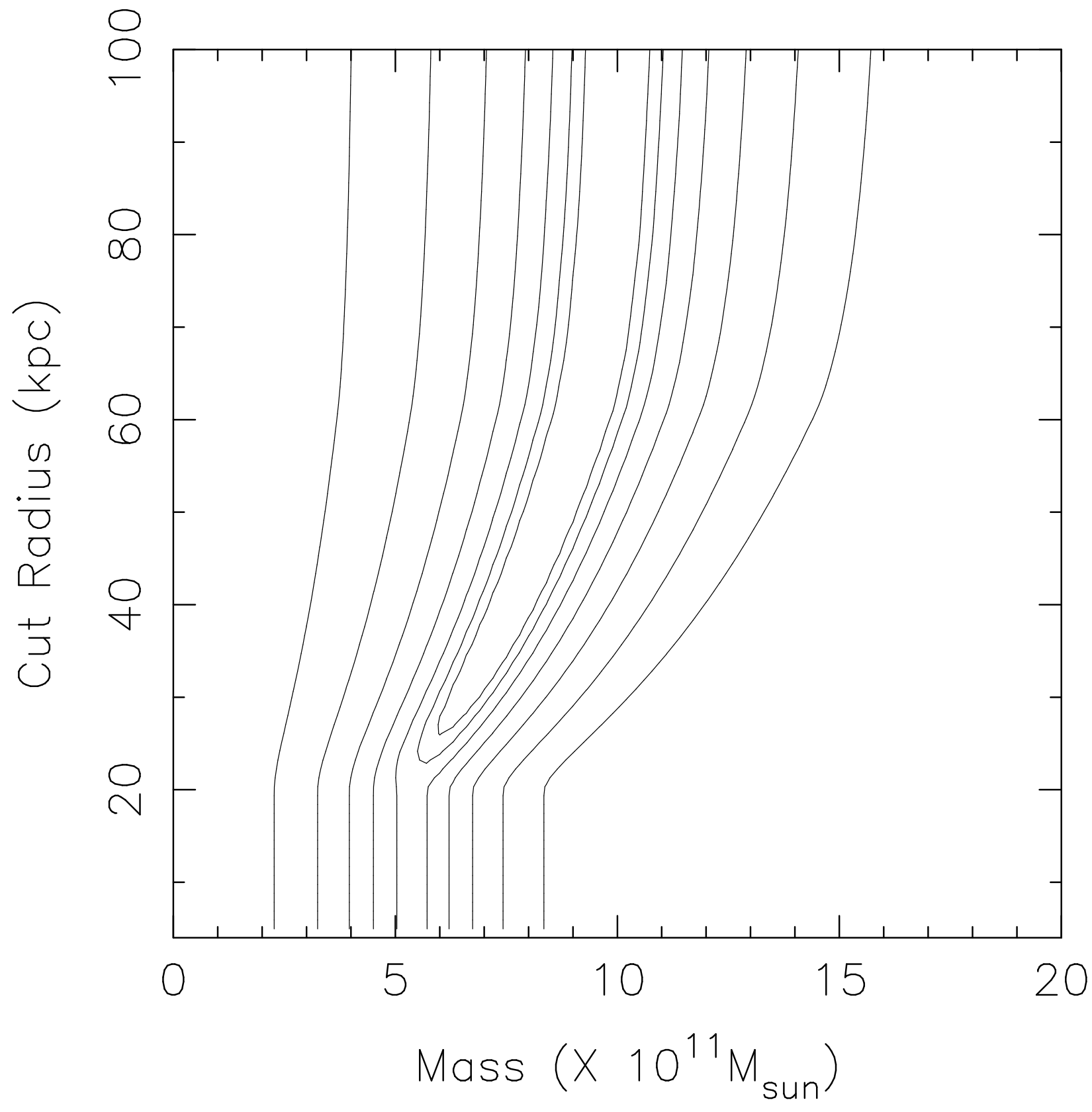

Fig. 3. $-\chi^{2}$ as a function of the mass $M_{o}\left(\right.$ in $\left.\times 10^{11} M_{\odot}\right)$ and the cut-off radius $r_{c}$ in kpc for the King galaxy profile. The solid contours denote $\chi^{2}=5,10,20,40,60,120$ and 240 . 


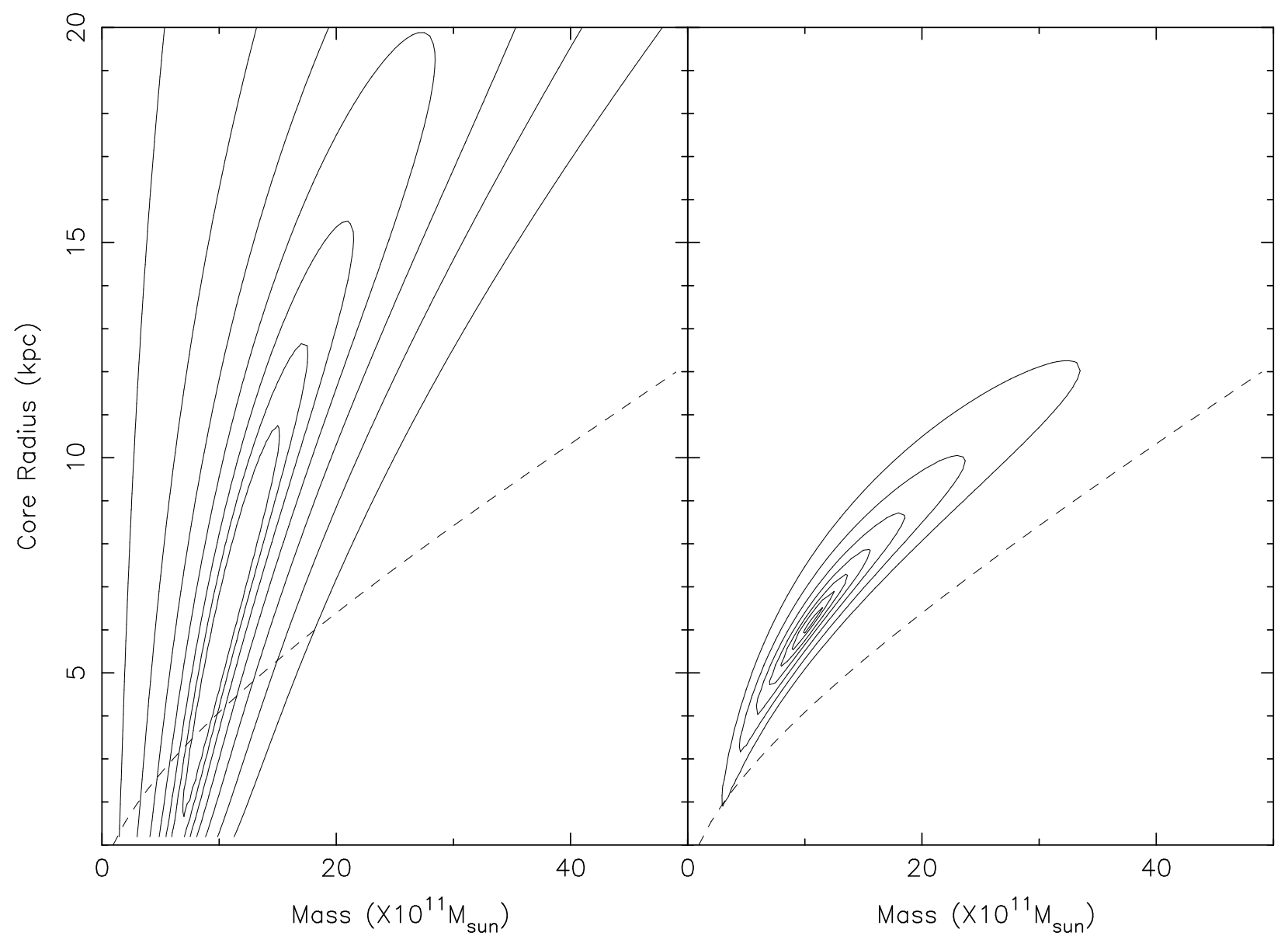

Fig. 4. $-\chi^{2}$ as a function of the mass $M_{o}\left(\right.$ in $\left.\times 10^{11} M_{\odot}\right)$ enclosed within a radius $R_{O}=60 \mathrm{kpc}$ and the core radius $a$ in $\mathrm{kpc}$ for the King profile. The solid contours denote $\chi^{2}=5,10,20,40,60,120$ and 240. The dashed line shows the minimum core radius such that there is no multiple imaging. The lens is placed at $5^{\prime \prime}$ (first panel) and $1^{\prime \prime}$ (second panel) from the center of the jet. 


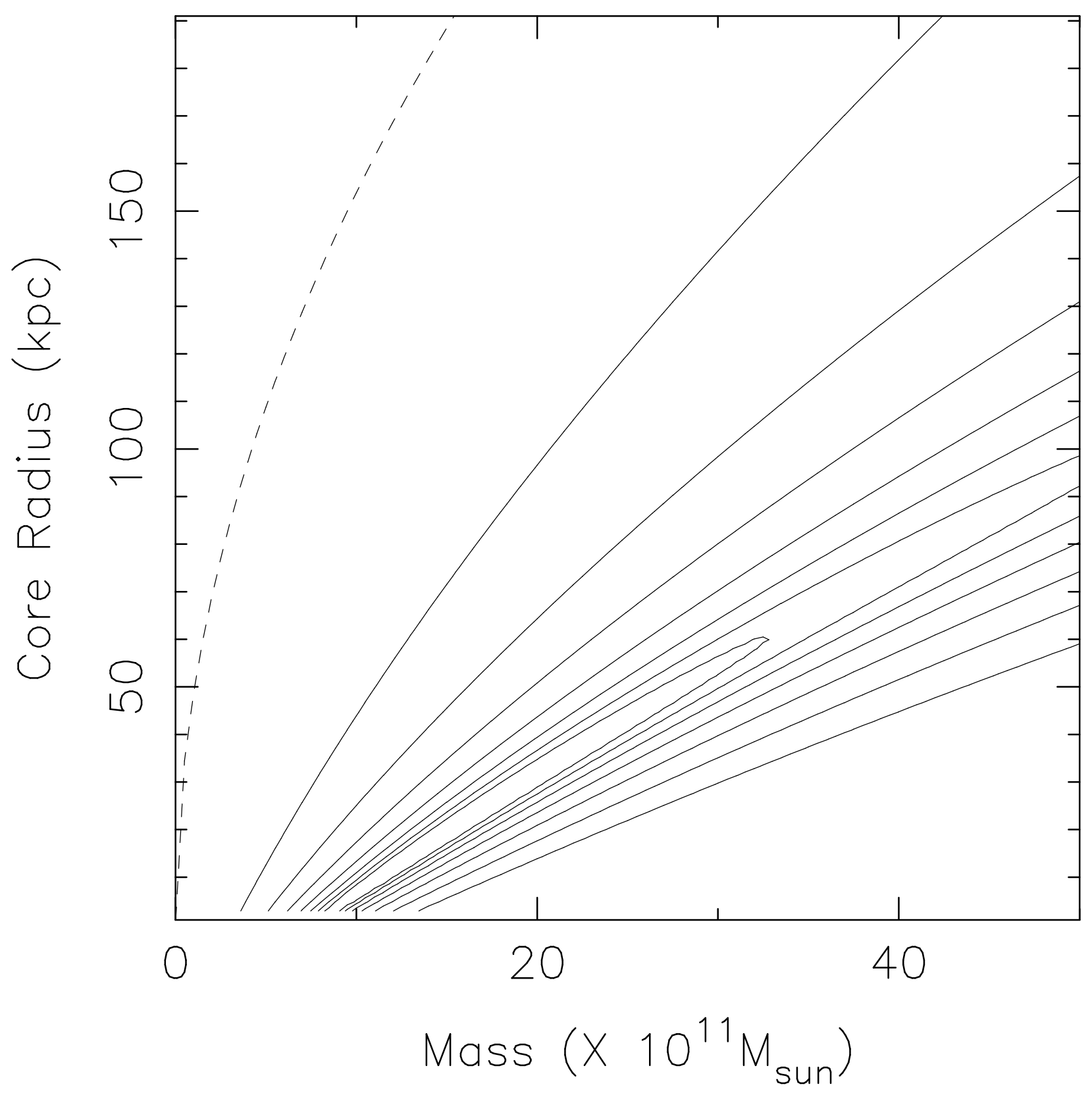

Fig. 5.- Same as Figure 4, except that a deVaucouleurs mass profile is used instead of a King profile. Note that all the $\chi^{2}$ contours are excluded by the single-image condition (dashed line). 

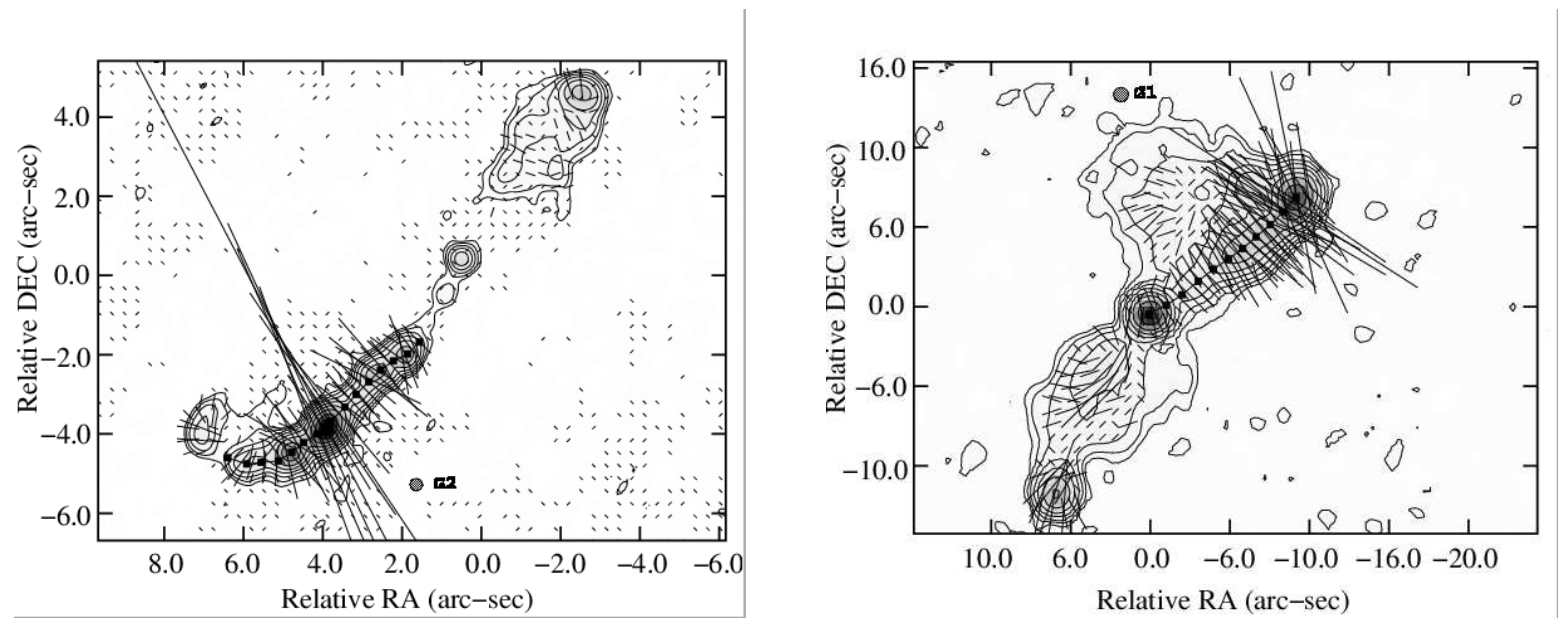

Fig. 6. - Radio contour map of quasar 3C9 at X-band and 1253+104 at C-band. Contours are drawn at $0.12,0.25,0.5,1,3,6,12,25$ and 50 percent of peak intensity, which is 56.5 mJy beam $^{-1}$ for 3c9 and $29.7 \mathrm{mJy} \mathrm{beam}^{-1}$. The magnetic field components of the polarization are drawn as vectors such that $1^{\prime \prime}$ is equivalent to a polarization intensity of $1 \mathrm{mJybeam}^{-1}$. The filled boxes connected by lines represent the fiducial line. 


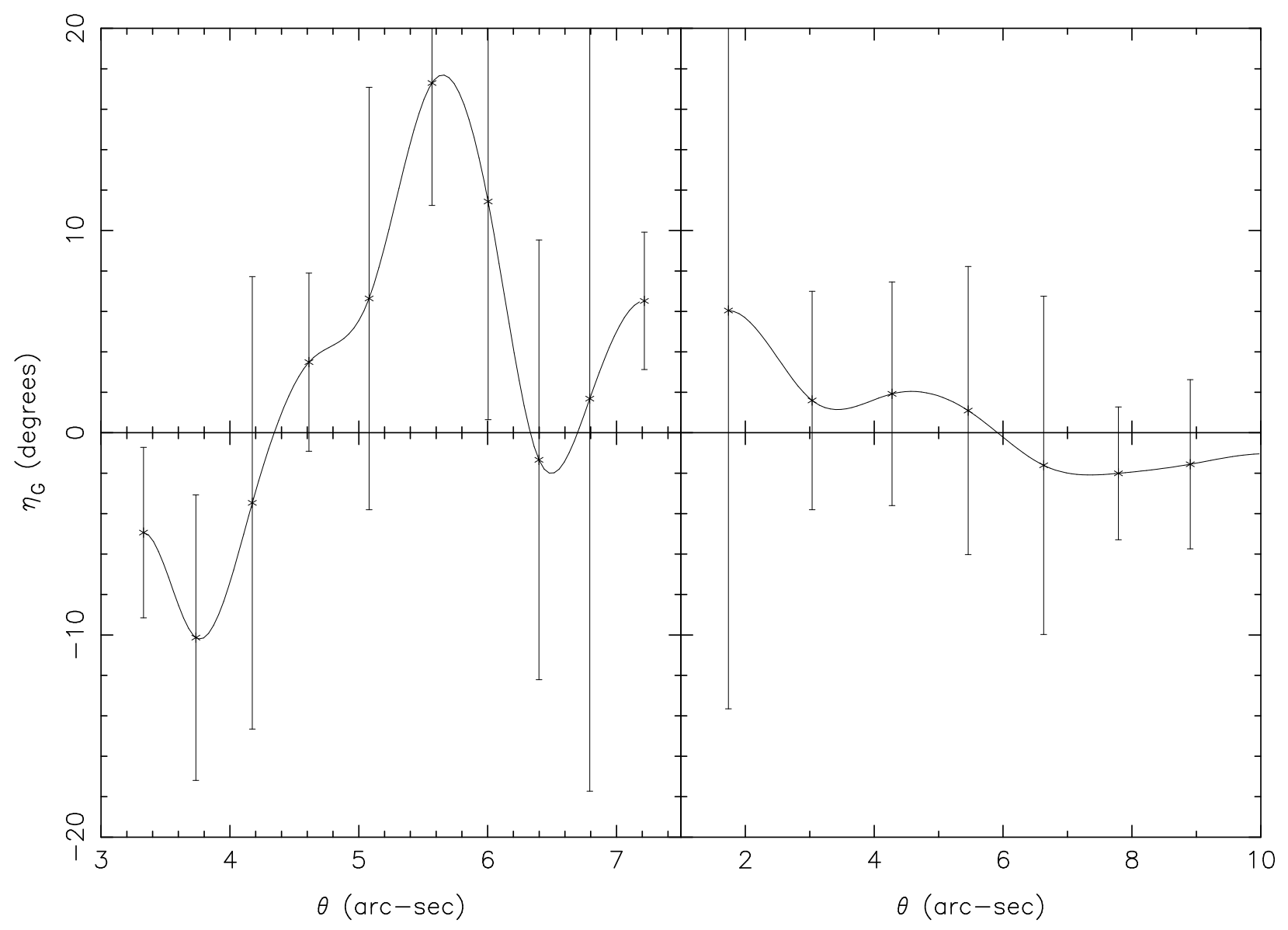

Fig. 7. - $\eta_{G}$ for the quasar 3C9 (first panel) and QSO 1253+104 (second panel). The horizontal axis shows $\theta$, the distance along the jet, in arc-seconds from the quasar center. The vertical axis shows $\eta_{G}$ in degrees. 

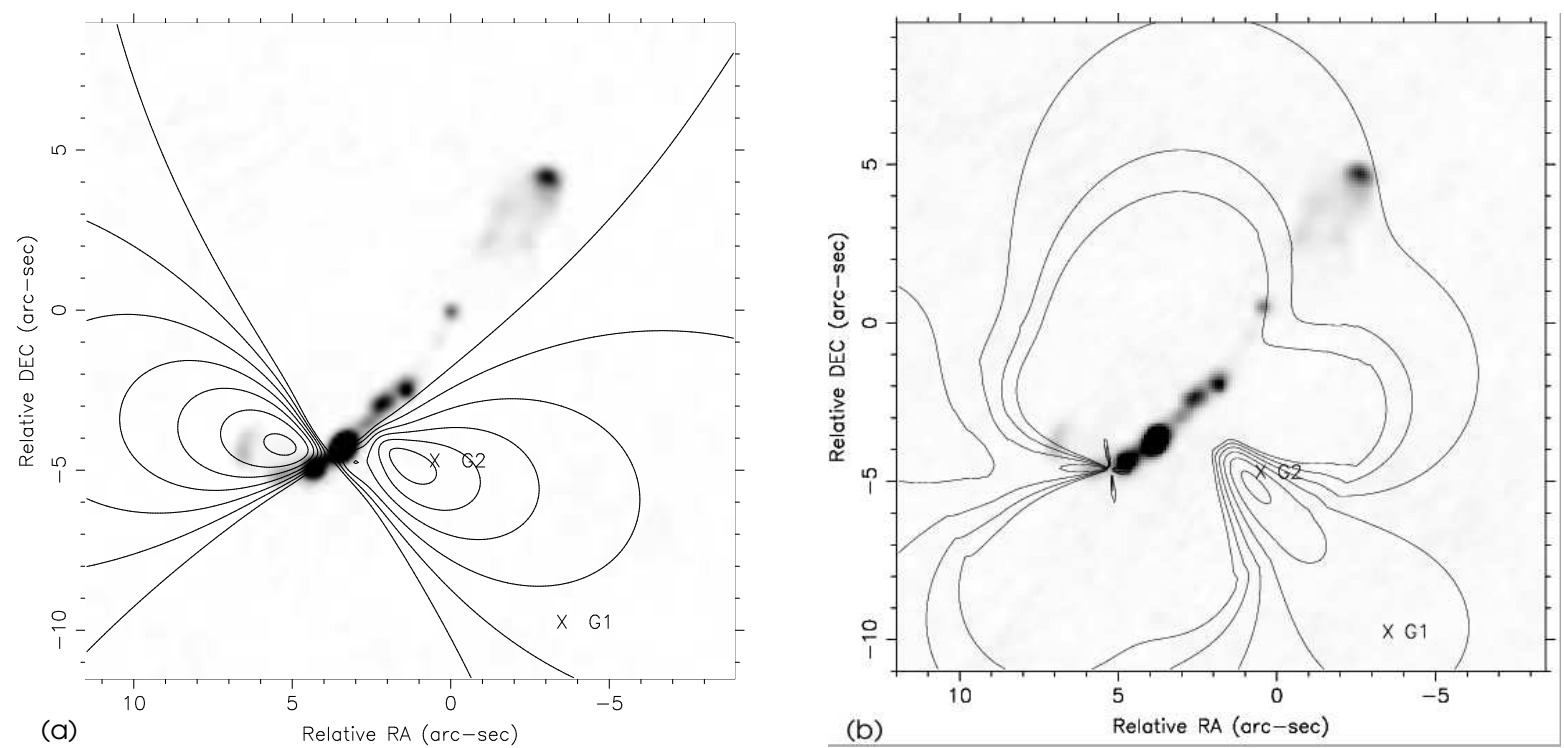

Fig. 8. - $\chi^{2}$ as a function of lens position on the sky for two different lens masses. The gray-scale image is of the quasar and jet, while the contours are of $\chi^{2}$. (a) The lens mass is $10 \times 10^{11} M_{\odot}$ and contours are drawn at $\chi^{2}=50,100,150,200,250,300$ and 350 . (b) The lens mass is $30 \times 10^{11} M_{\odot}$ and contours are drawn at $\chi^{2}=5,10,15,20,25$ and 30. Galaxies G1 and G2 (as denoted in (Kronberg et al. 1996)) are labeled for reference. In both cases, the lens redshift is 1.0, the core radius is $2 \mathrm{kpc}$ and the cut radius is $30 \mathrm{kpc}$. 

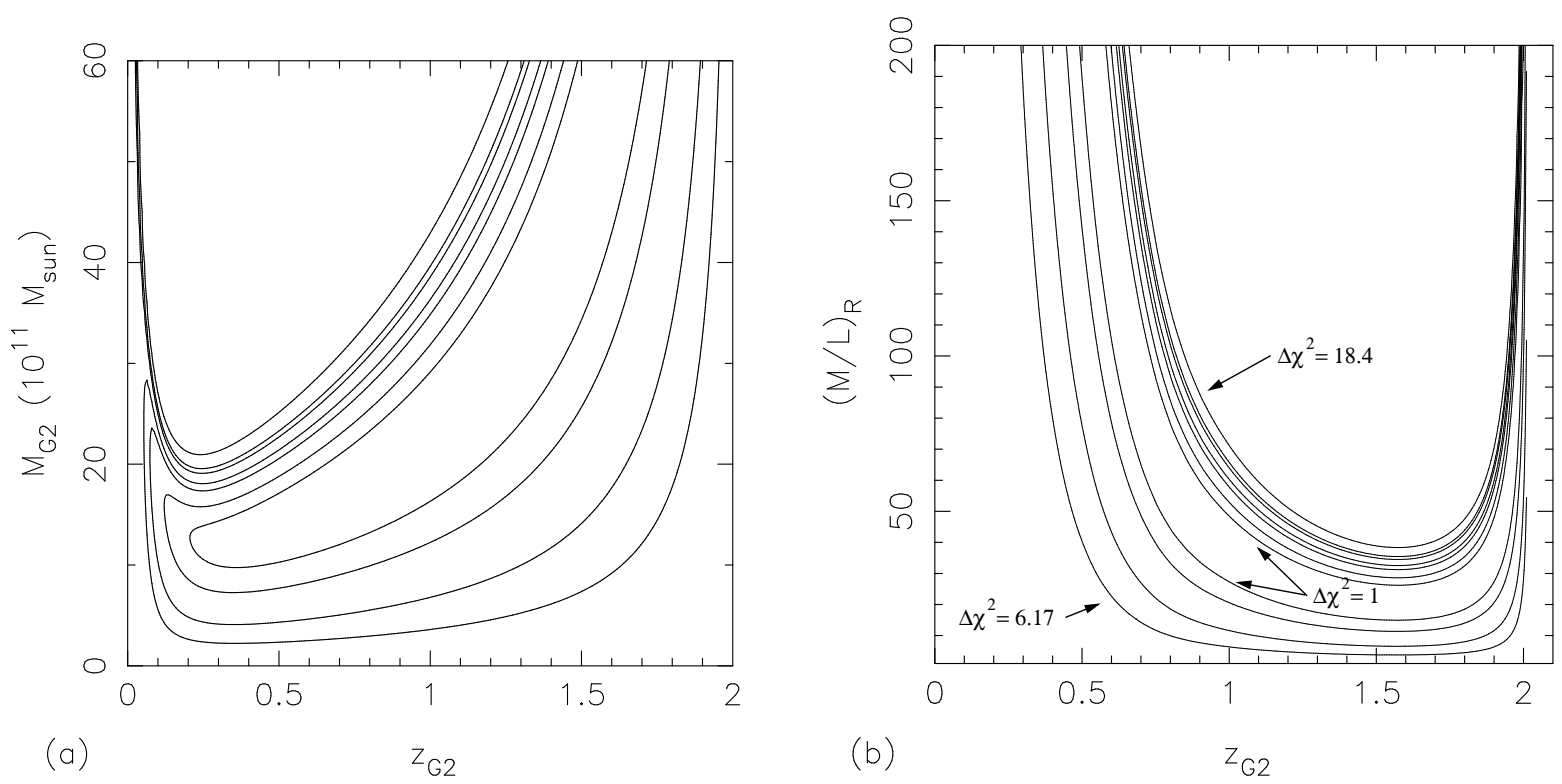

Fig. 9.- (a) $\chi^{2}$ as a function of redshift and mass of lens G2. (b) $\chi^{2}$ as a function of redshift and $\mathcal{M} / \mathcal{L}$ for lens $\mathrm{G} 2$. The minimum $\chi^{2}$ is 3.6 and contours are drawn at $\Delta \chi^{2}=$ 1.0,2.3, 4.6,6.17,9.21, 11.0 and 18.4. In both cases, the core and cut radii are $5 \mathrm{kpc}$ and 30 kpc, respectively, and the mass of G1 is held at $17 \times 10^{11} M_{\odot}$.
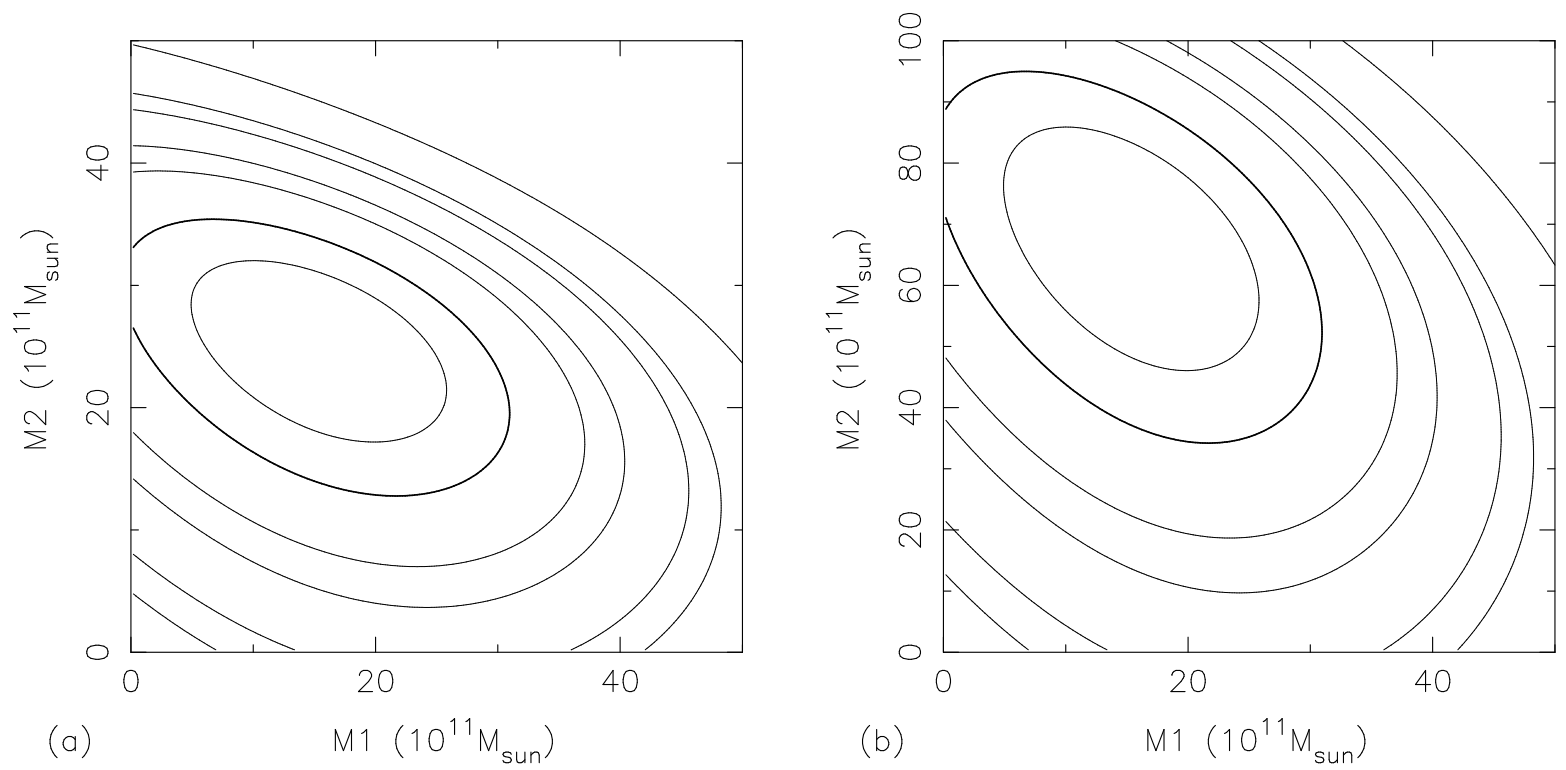

Fig. 10. - $\chi^{2}$ as a function of the masses of G1 and G2 for two different redshifts of G2: (a) $z_{2}=1.0$ and (b) $z_{2}=1.6$. Contours are drawn at the same levels as figure 9 . The $\Delta \chi^{2}=2.3$ contour is taken as the 1- $\sigma$ confidence region for the joint probability of G1 and G2 and is drawn as a bold line. In both cases, core and cut radii for the two lenses are $5 \mathrm{kpc}$ and $30 \mathrm{kpc}$, respectively. 


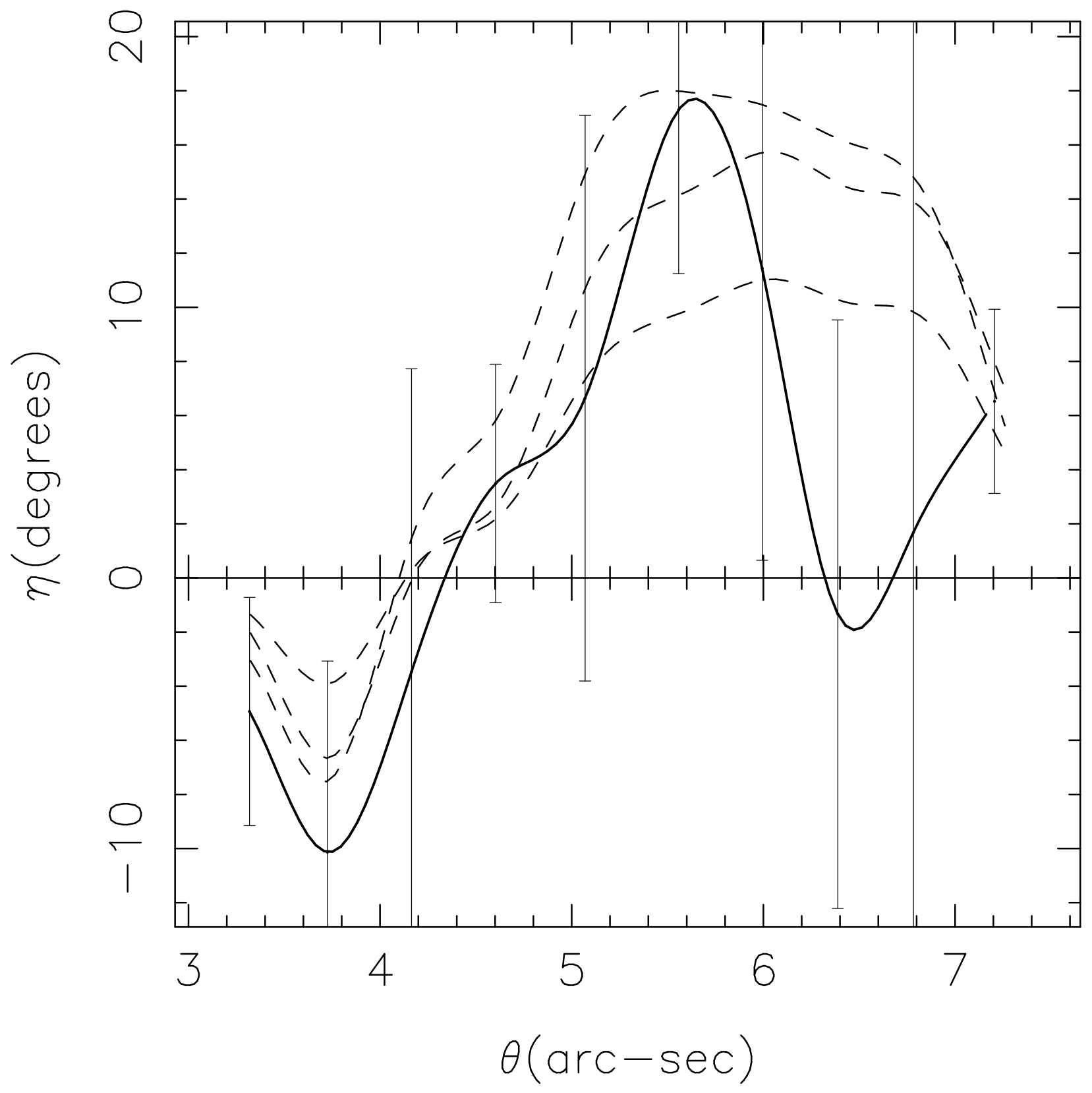

Fig. 11. - Best fit $\eta_{G}$ assuming $z_{2}=1.0, M_{G 2}=3 \times 10^{12} M_{\odot}$ and $M_{G 1}=1.7 \times 10^{12} M_{\odot}$. The observed data (solid line) and three representative fits are plotted (dashed lines). The middle dashed curve is the best fit and the two outer curves correspond to 1- $\sigma$ fits. The core and cut radii are $5 \mathrm{kpc}$ and $30 \mathrm{kpc}$, respectively, for both lenses. 


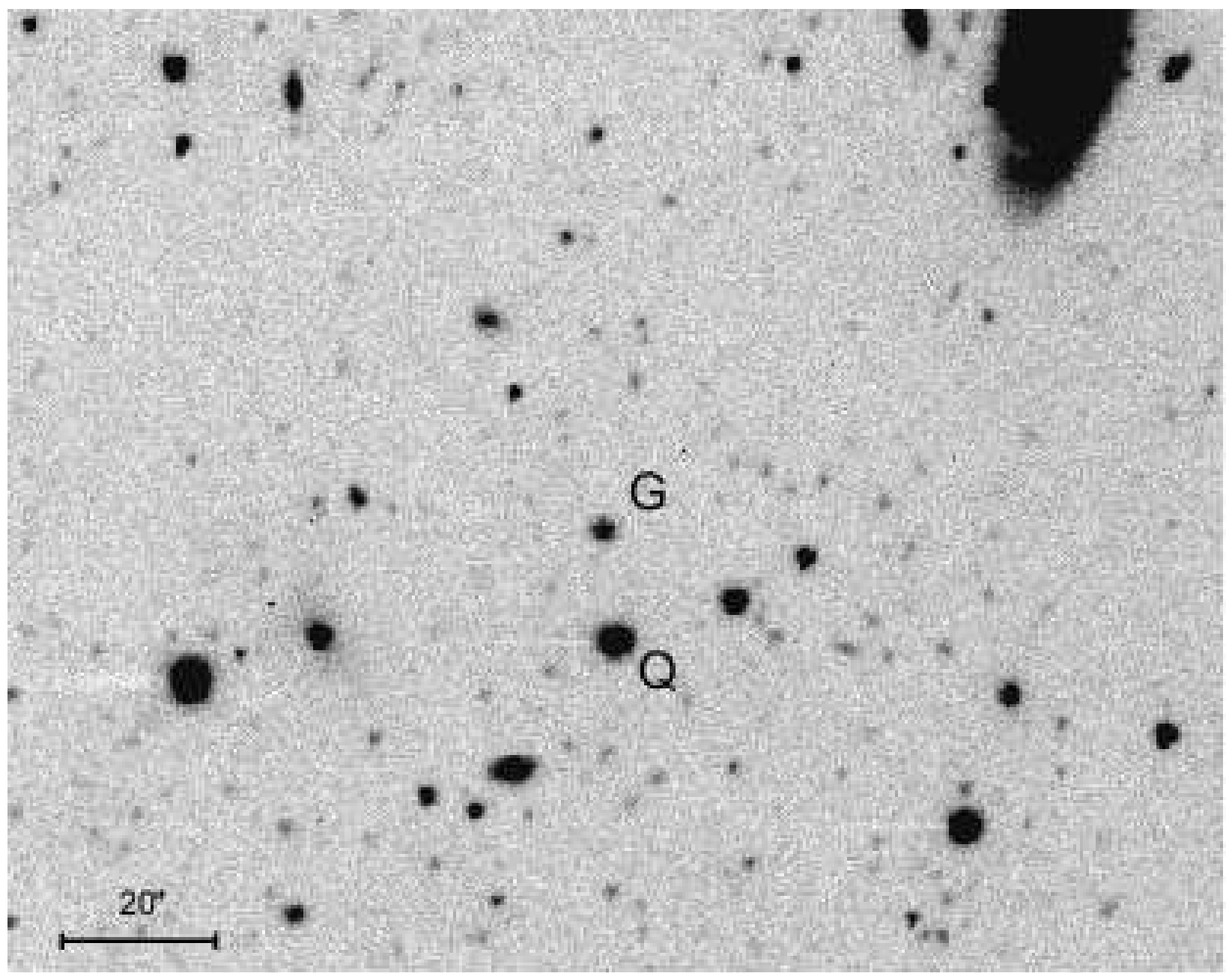

Fig. 12. - Sum of three $600 \mathrm{sec}$ exposures of the field of Q1253+104 in the R-band. The quasar is labelled Q and the galaxy with G. North is up and East to the left. 


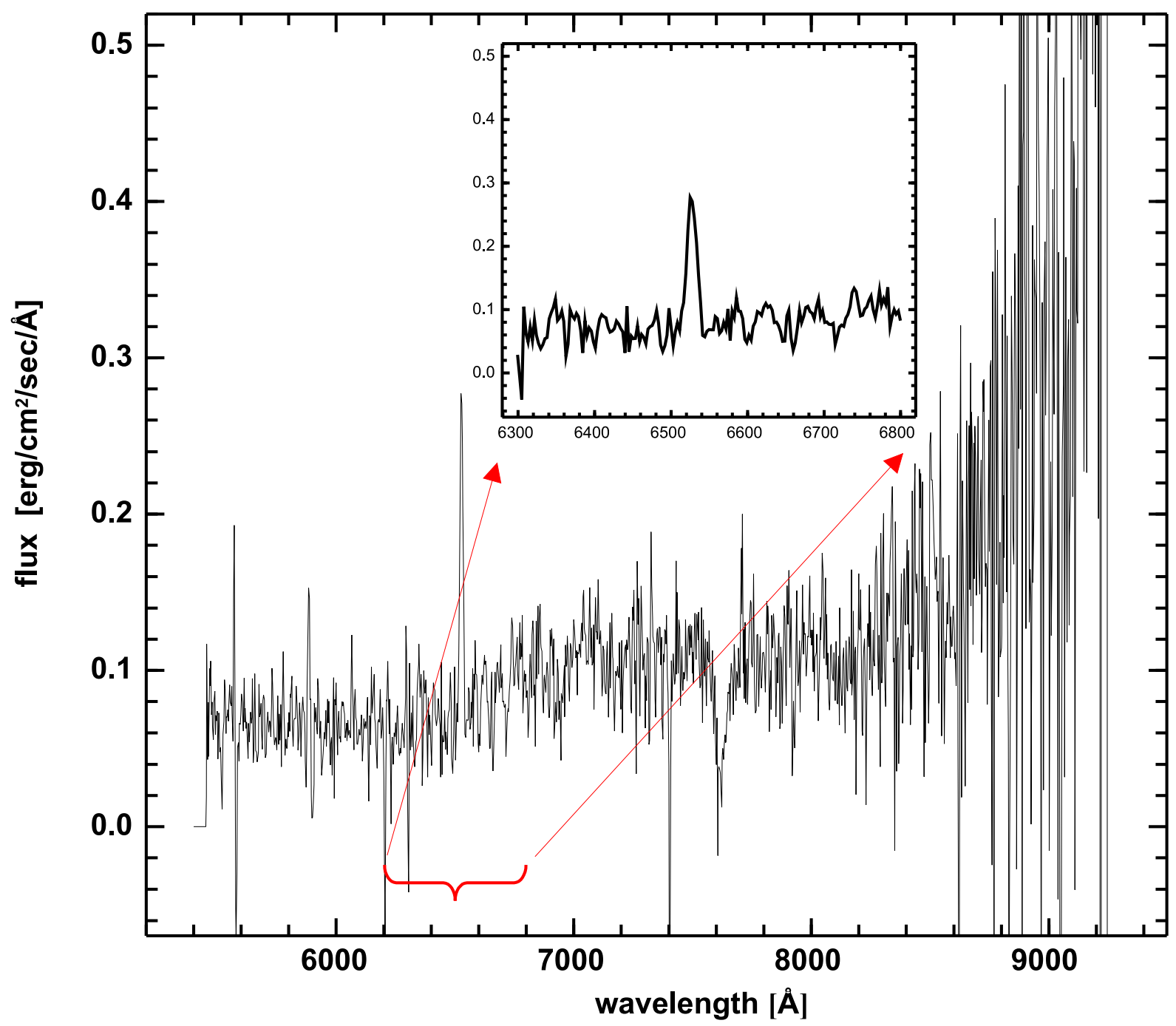

Fig. 13. - Spectrum of galaxy G north of quasar Q1253+104 showing a single prominent emission line at $6527 \AA$. If this line is due to [OII] 3727 the redshift of the galaxy is 0.75 . 


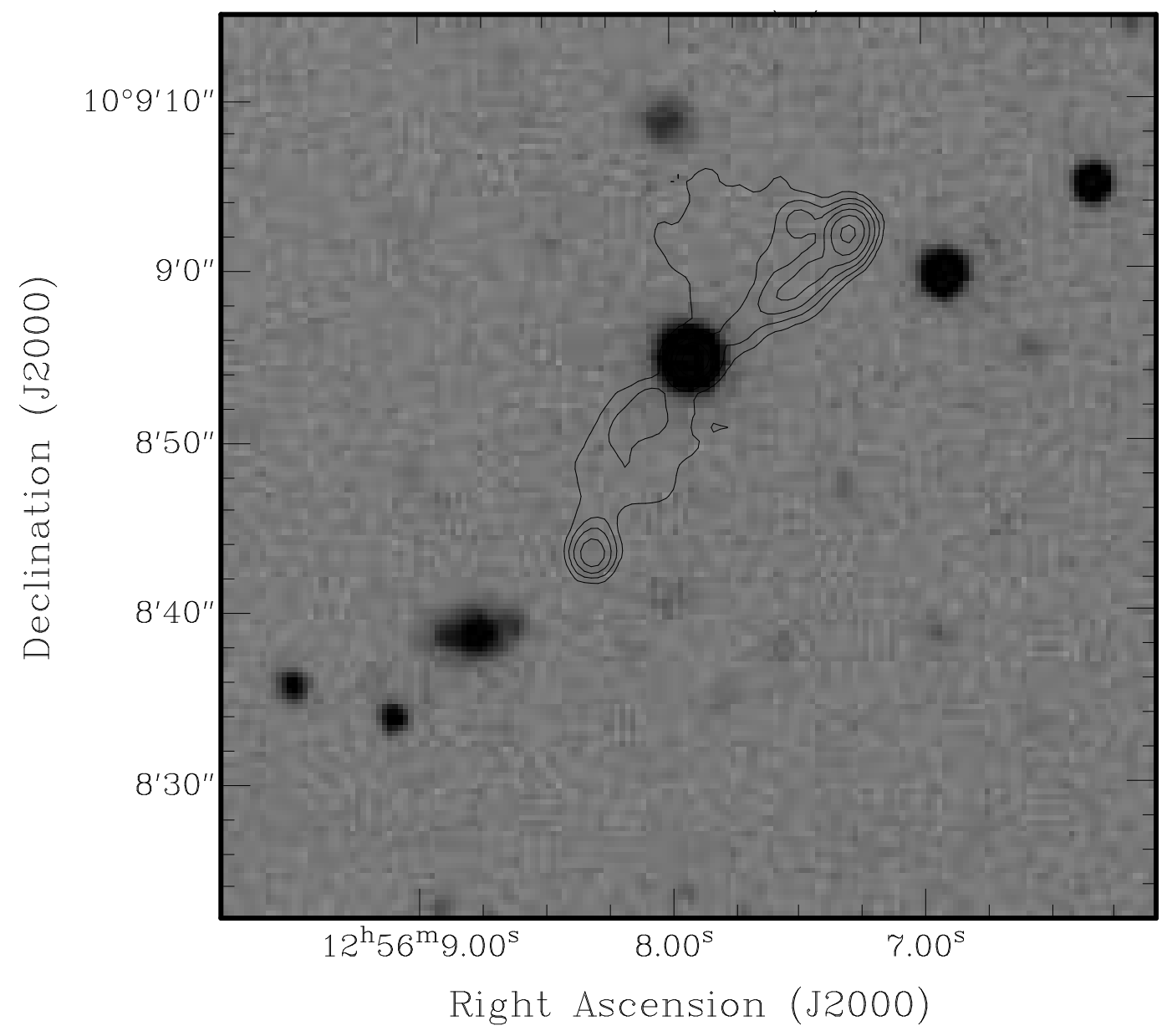

Fig. 14. - The optical field of $1253+104$ in R (left) and B (right) bands, taken using the $3.5 \mathrm{~m}$ telescope at the Calar Alto observatory. Spectroscopy of the north-east galaxy places it at a redshift of 0.75 . The contours are of the VLA C-band radio observations. 
Table 1. Derived parameters for the 3C9 lenses.

\begin{tabular}{lccccl}
\hline \hline Assumed $z_{2}$ & $M_{G 1}$ & $(\mathcal{M} / \mathcal{L})_{G 1}$ & $M_{G 2}$ & $(\mathcal{M} / \mathcal{L})_{G 2}$ & $a_{G 2}$ \\
& $10^{11} M_{\odot}$ & $M_{\odot} / L_{\odot}$ & $10^{11} M_{\odot}$ & $M_{\odot} / L_{\odot}$ & $\mathrm{kpc}$ \\
\hline 1.0 & $17 \pm 15$ & $<75^{\mathrm{a}}$ & $25 \pm 10$ & $\lesssim 40$ & $>5$ \\
1.6 & $17 \pm 15$ & $<75^{\mathrm{a}}$ & $65 \pm 15$ & $\lesssim 30$ & $>5$ \\
$0<z_{2}<2$ & $17 \pm 10$ & $<75^{\mathrm{a}}$ & $\gtrsim 10$ & $\gtrsim 20$ & $\cdots$ \\
\hline
\end{tabular}

aTaken from Kronberg et al. (1996). 